\title{
Análisis temporal del riesgo por malformaciones congénitas atribuibles al uso de plaguicidas en el corredor florícola del Estado de México
}

\author{
Rosa Estela Hernández Valdés*, Miguel Ángel Gómez Albores*, Alejandro Tonatiuh Romero Contreras*, Marcela Virginia \\ Santana Juárez*, Carlos Alberto Mastachi Loza*, Marivel Hernández Téllez* y Héctor Martínez Valdés*
}

Recepción: 4 de mayo de 2016

Aceptación: 8 de noviembre de 2016

*Universidad Autónoma del Estado de México, México. Correos electrónicos: stellahgo@yahoo.com.mx; magomeza@uaemex.mx; lautona@aol.com; mvsantana7@hotmail.com; mastachii@hotmail.com; marivelht@yahoo.com.mx; hmartinezv@uaemex.mx Se agradecen los comentarios de los árbitros de la revista.

(c) $\$=$
Resumen. Se analizó el comportamiento temporal (1998-2013) de las tasas de mortalidad fetal e infantil de anencefalia $(\mathrm{Q} 00)$ y espina bífida (Q05), cuya etiología puede derivar de la exposición a los plaguicidas. Se compararon los municipios del corredor florícola del Estado de México de mayor actividad agrícola con aquellos de menor actividad, a través del uso de medidas de asociación, bajo un estudio de tipo ecológico retrospectivo. Los resultados indican una posible asociación con un riesgo de defunciones fetales por anencefalia más alto en las zonas expuestas (OR 3.4; IC 95\% 2.72-4.27; AR $65.2 \%$ ), asimismo para la mortalidad infantil por espina bífida (OR 7.1; IC 95\% 4.51-11.12; AR 79.8\%), ambos con $p<0.05$ utilizando la prueba exacta de Fisher.

Palabras clave: anencefalia, espina-bífida, corredor florícola, plaguicida, riesgo-atribuible.
Temporal Analysis of Risk of Congenital Malformations Attributable to the Use of Pesticides in the Floriculture Corridor from the State of Mexico

Abstract. The temporal behavior (19982013) of the rates of fetal and child mortality by anencephaly $(\mathrm{Q} 00)$ and spina bifida $(\mathrm{Q} 05)$ which etiology may be derived from de exposure of pesticide, was analyzed and compared between the municipalities of the floriculture corridor from the State of Mexico with high agricultural activity and those with minor activity, through the use of measures of association; under a retrospective ecological study.

The results shown a possible association with a higher risk of fetal dead by anencephaly in the exposed areas (OR 3.4; IC 95\% 2.72-4.27; AR $65.2 \%$ ), likewise for infant mortality by spina bifida (OR 7.1; IC 95\% 4.51-11.12; AR $79.8 \%$ ) both, with a $p<0.05$ using the exact Fisher's test.

Keywords: anencephaly, spina-bifida, floriculture corridor, pesticide, risk-attributable.

\section{Introducción}

En México el cultivo de plantas ornamentales es importante para el sector agrícola en términos del comercio nacional e internacional. Para 2012, éste sector alcanzó una superficie sembrada de 20 mil ha, de las cuales se estima que $70 \%$ de la distribución, producción y consumo es de flores y plantas. En sólo cuatro estados se registra 87.2\% de producción total. El primer lugar lo ocupa el Estado de México con 61.3\% del total nacional, seguido de Puebla con 14.5\%, Morelos con 7.6\% y el Distrito Federal con 3.8\%. En orden del valor de la producción destacan las rosas, crisantemos, gladiolas y 
nochebuenas, en la mayoría de los casos con tecnificación (riego y agroquímicos) (FND, 2014).

Los municipios del Estado de México más importantes en los que se desarrolla la actividad florícola son Villa Guerrero, Coatepec Harinas, Tenancingo, Zumpahuacán, Malinalco e Ixtapan de la Sal, en los cuales se encuentra concentrado $90 \%$ de la producción estatal y $80 \%$ de la producción nacional de los principales cultivos de flor. Villa Guerrero por si solo genera $56 \%$ de la producción total estatal (Sánchez y Pacheco, 2009).

En la actividad florícola prevalece un elevado uso de plaguicidas, tanto en campo (sistema abierto) como en invernaderos (sistema cerrado). Dicho uso se ha asociado a riesgos en la salud de las personas expuestas, especialmente en niños y mujeres embarazadas, que apoyan las actividades de fumigación, corte y acarreo de las flores (Oviedo-Zuñiga, 2013).

Se han llevado a cabo diferentes estudios en los que se ha encontrado una relación entre el uso de plaguicidas y las afectaciones al sistema nervioso, endocrino, digestivo, inmunológico y reproductivo (Plengue-Telechea et al., 2007; Espitia-Rangel et al., 2003; Goldman, 1997; Ramírez-Espitia et al., 2003). De forma específica se ha relacionado la exposición ocupacional de los padres a los plaguicidas con la presencia de malformaciones congénitas como espina bífida y anencefalia (Benítez-Leite et al., 2009; Chen, 2008). Cabe mencionar que ambas tienen una etiología multifactorial, es decir, por diversos factores genéticos o ambientales; sin embargo, la actividad florícola y la exposición a plaguicidas es un factor que contribuye a su presencia (CEVECE, 2014).

Otros estudios también han encontrado una asociación positiva entre exposición a plaguicidas y los efectos que causa en la salud, tal es el caso de la Systematic Review of Pesticide Health Effects que relaciona el desarrollo fetal y los defectos del tubo neural con la exposición a plaguicidas (Samborn et al., 2012), por lo cual destaca un mayor riesgo para anencefalia (Stemp-Morlock, 2007; Muñoz Piña y Ávila Forcada, 2005) y para espina bífida, aunque en menor orden de importancia (Blater et al., 2000; Brender et al., 2010).

Los plaguicidas aplicados comúnmente en México son los organofosforados, carbamatos y organoclorados, todos con efectos tóxicos en el sistema nervioso (Leyva-Morales et al., 2014; Hernández-Antonio y Hansen, 2011; González-Arias et al., 2010; Karam et al, 2004). Por tal motivo se ha hecho énfasis en las políticas de salud pública para evitar o disminuir el uso de los más peligrosos, aunque no se ha obtenido el éxito deseado (Muñoz Piña y Ávila Forcada, 2005; KaramQuiñones, 2002).

México ha experimentado un aumento de muertes por defectos de nacimiento, como los de cierre del tubo neural, en el que se incluyen la anencefalia y espina bífida. En este contexto, el porcentaje de defunciones aumentó de $4 \%$ en 1979 a $18.3 \%$ en el 2003 a nivel nacional. Los estados de la República Mexicana que más casos presentaron de 1998 al 2003 son el Estado de México, seguido por el Distrito Federal y el estado de Puebla (INEgi, 2013; Gómez-Alcalá y Rascón Pacheco, 2008; Mancebo-Hernández et al., 2008; Peña-Alonso, 2005). La situación que coincide con los estados de mayor producción florícola.

Por lo tanto, el objetivo del trabajo se centra en determinar mediante un estudio de tipo ecológico-retrospectivo si existe una posible asociación entre el uso de plaguicidas y la mortalidad fetal e infantil por anencefalia y espina bífida en una zona florícola de importancia comercial en México. El estudio contempló el cálculo de tasas de mortalidad anual para medir la frecuencia y la razón de riesgo (odss ratio, OR) y atribuible como medidas de asociación.

\section{Materiales y método}

El corredor florícola en el sur del Estado de México especializado en flor de corte, cultivo de hortalizas y huertas lo integran los municipios de Almoloya de Alquisiras, Coatepec Harinas, Ixtapan de la Sal, Malinalco, Tenancingo, Tonatico, Villa Guerrero y Zumpahuacán (mapa 1).

Las condiciones climáticas en el sur del Estado de México han favorecido la formación de un corredor natural para la producción de flor de corte, así como de hortalizas y huertas, cultivadas tanto en campo como en invernaderos, con un clima que va del templado subhúmedo en la parte alta al semicálido en la parte baja, con temperatura media anual entre $\operatorname{los} 13$ y $24^{\circ} \mathrm{C}$, y una precipitación total anual entre 501 a

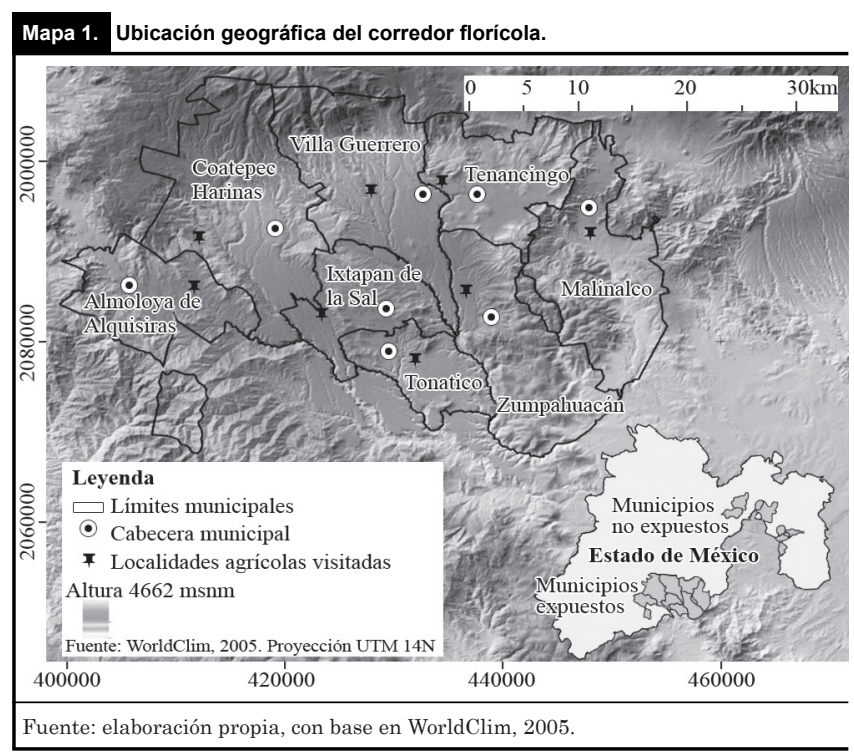


$1500 \mathrm{~mm}$ (WorldClim, 2005). La superficie total de los municipios que integran el corredor es de $1648 \mathrm{~km}^{2}$, de los cuales 41.1\% corresponde a uso de suelo agrícola (INEGI, 2011).

El corredor se encuentra dentro de la cuenca del Alto Amacuzac, donde existe un sistema montañoso que comienza desde la ladera sur del volcán Nevado de Toluca, y desciende desde Villa Guerrero a 3900 msnm hasta una mínima de 1 660 msnm en el municipio de Zumpahuacán. La dirección de sus vientos y escurrimientos de agua superficial y subterránea va de norte a sur, de las partes altas con un suelo de origen ígneo a las bajas con un suelo de origen sedimentario de mayor permeabilidad y capacidad de filtración. Los municipios con mayor número de unidades de producción, invernaderos, ventas y antigüedad en el uso de plaguicidas son Villa Guerrero, Tenancingo y Coatepec Harinas (INEGI, 2009; INEGI, 2010).

La población total en los municipios del corredor florícola es de 289596 habitantes, de los cuales 64\% se concentra en Tenancingo, Villa Guerrero y Coatepec Harinas. La fuerza de producción que aplica plaguicidas es mayormente masculina (67\% en el caso de los familiares que participan en la floricultura y $90 \%$ de los contratados), y fluctúa entre edades de 18 a 60 años. Sin embargo, es muy común que los familiares principalmente mujeres y niños menores de 12 años se involucren en la actividad (INEGI, 2009; INEGI, 2010).

Dada la información disponible de defunciones totales y fetales proporcionadas por la Dirección General de Información en Salud (DGIS), la investigación se enmarca en un estudio de tipo ecológico en el que la unidad de análisis no es el individuo, sino las mediciones agregadas a los municipios; asimismo, retrospectivo dado que se analiza el efecto del evento para conocer sus causas, longitudinal porque considera un lapso en el tiempo y pseudoanalítico, ya que su fin es evaluar una relación causal entre un factor y un efecto para conglomerados de población. Así pues, los resultados generados permitirán sugerir relaciones hipotéticas de causa-

Cuadro 1. Población dedicada a la agricultura.
\begin{tabular}{|lclc}
\hline Municipios del corredor & $\begin{array}{c}\text { Porcentaje de } \\
\text { trabajadores agrícolas }\end{array}$ & \multicolumn{1}{c}{$\begin{array}{c}\text { Municipios con menor } \\
\text { actividad agrícola }\end{array}$} & $\begin{array}{c}\text { Porcentaje de } \\
\text { trabajadores agrícolas }\end{array}$ \\
\hline Almoloya de Alquisiras & 39.34 & Atizapán de Zaragoza & 0.14 \\
Coatepec Harinas & 45.43 & Coacalco de Berriozábal & 0.14 \\
Ixtapan de la Sal & 17.79 & Chicoloapan & 0.35 \\
Malinalco & 29.62 & Chimalhuacán & 0.32 \\
Tenancingo & 27.02 & Ecatepec de Morelos & 0.11 \\
Tonatico & 26.50 & La Paz & 0.12 \\
Villa Guerrero & 35.97 & Cuautitlán Izcalli & 0.34 \\
Zumpahuacán & 55.79 & Valle de Chalco Solidaridad & 0.25 \\
\hline Fuente: elaboración con datos del INEGI, 2013. & & \\
\hline
\end{tabular}

lidad (Argimon, 2013; Hernández, 2007; Hernández-Ávila et al., 2000).

El estudio consistió en recorridos de campo in situ, donde se aplicó el método antropológico de observación directa y registro con diario de campo (Viqueira et al., 1971). Se tomaron notas detalladas acompañadas de fotografías sobre las prácticas y conductas culturales asociadas con la aplicación de plaguicidas en los cultivos, tanto en campo como en invernaderos. Se realizaron 45 visitas periódicas a las localidades con mayor superficie de terreno cultivado y mayor antigüedad en el proceso de producción de los ocho municipios. Entre noviembre 2013 y abril 2015, el objetivo fue identificar la exposición y contacto con plaguicidas en la población trabajadora, así como saber cuál se aplicó con mayor frecuencia, el grupo químico y su grado de toxicidad. Esto se logró a través de la información obtenida de entrevistas semiestructuradas, donde 24 productores agrícolas participaron de forma voluntaria y de la observación directa en campo.

Posteriormente se identificaron dos enfermedades para relacionarlas con la exposición y contacto con plaguicidas. La primera: la anencefalia, que es un defecto por la falla del neuroporo cefálico para cerrarse de manera adecuada, la cual se caracteriza por la ausencia de huesos del cráneo, cuero cabelludo y presencia de un encéfalo rudimentario. La segunda: la espina bífida, que se refiere al cierre inadecuado del neuroporo posterior y se manifiesta como una falta de fusión de los arcos vertebrales (DGE, 2012). Ambas corresponden a defectos del tubo neural, también consideradas como malformaciones congénitas (OMS, 2007).

Para facilitar el cálculo de indicadores de frecuencia y de asociación, las bases de datos (BD) de defunciones fetales y nacimientos, así como la mortalidad y población infantil (SINAIS, 2016a; SINAIS, 2016b; INEGI, 2010), se procesaron en Microsoft Access. Cabe mencionar que para la selección de las causas se utilizó la Clave Internacional de Enfermedades CIE-10, Q00 para anencefalia y Q05 para espina bífida (OPS, 2008; y OMS, 2008).

Como medidas de frecuencia se calcularon las tasas de mortalidad fetal e infantil para compararlas entre los municipios con mayor y menor exposición a plaguicidas, tanto para el corredor florícola, donde de 17 a $56 \%$ de la población está dedicado a la agricultura, como para los municipios donde la actividad ocupacional de la población en la agricultura es menor a $1 \%$ (cuadro 1 ). 
Para los indicadores de frecuencia se calculó las tasas de mortalidad fetal (TMF) para medir la probabilidad de morir antes del nacimiento sin importar el periodo de gestación. La forma de cálculo fue la siguiente:

$T M F=\frac{\mathrm{N}^{\circ} \text { muertes fetales en un año }}{\mathrm{N}^{\circ} \text { nacidos totales en ese mismo año }} x F$ (vivos y muertos)

Donde: $F=$ factor de ajuste. Equivalente a 1000 habitantes para este caso.

El otro indicador de frecuencia fue la tasa de mortalidad infantil (TMI) para medir la probabilidad de morir que tiene un nacido vivo antes de cumplir un año de edad (MorenoAltamirano, 2000). Se calcula con la ecuación:

$T M I=\frac{\mathrm{N}^{\circ} \text { muertes de menores de } 1 \text { año de edad en un año }}{\mathrm{N}^{\circ} \text { nacidos vivos registrados en ese mismo año }} \times F$

Para los indicadores de asociación se calculó la razón de momios (OR, por sus siglas en inglés) por medio de la obtención del cociente de los productos cruzados de una tabla $2 \times 2$, que es la medida para conocer la probabilidad de padecer una enfermedad o morir en función de la exposición (Jewell, 2004; Hernández, 2009). Es así que para una causa $D$, asociada con un factor de exposición $E$, el or se obtiene:

$O R=\frac{P(D \mid E)}{P(\text { No } D \mid E)} / \frac{P(D \mid N o E)}{P(\text { No } D \mid N o E)}$

Donde: $P(D \mid E)=$ probabilidad de morir en expuestos; $P($ No $D \mid E)=$ probabilidad de no morir en expuestos; $P$ $(D \mid$ No $E)=$ probabilidad de morir en no expuestos y $P$ (No $D \mid$ No $E)$ = probabilidad de no morir en no expuestos. Asimismo se calculó con paquete estadístico sPSs (Statistical Package for the Social Science) versión 22, el intervalo de confianza (IC) a 95\% y la prueba exacta de Fisher para medir la significancia estadística.

El segundo indicador de asociación fue el riesgo atribuible poblacional porcentual ( $\mathrm{RAP} \%)$, medida que permitió estimar la proporción de enfermedad en la población y que se explica por la exposición al factor estudiado. La forma de cálculo es:

$R A P \%=\frac{T M_{E X P}-T M_{N O E X P}}{T M_{E X P}}$
Donde: $T M_{E X P}=$ tasa de mortalidad de la población expuesta y $T M_{N O} E X P=$ tasa de mortalidad de la población no expuesta.

\section{Resultados y discusión}

Entre los principales cultivos de flores se encontraron las rosas, claveles, lilis, polares y follajes. En el cultivo de hortalizas predominó el jitomate, chile manzano y jalapeño, así como pepino, calabazas y fresas, además de huertas de duraznos, guayabas, aguacates y caña de azúcar. Todos los cultivos especializados y cultivados bajo paquetes tecnológicos con uso intensivo de plaguicidas por trabajadores locales (Herrera Tapia, 2006).

De las respuestas de los 24 productores entrevistados, así como de lo que se observó en los cultivos, se identificaron 51 tipos de plaguicidas aplicados con mayor frecuencia (32 insecticidas, 16 fungicidas y 3 herbicidas), de los cuales $29 \%$ corresponde a compuestos organofosforados. Por otra parte, se reconoció una lista de 34 ingredientes activos que se siguen empleando aun cuando han sido prohibidos o señalados como altamente peligrosos a nivel internacional, por los efectos nocivos a la salud y al ecosistema, como el carbofurán, metamidofos, monocrotofos, propargite y paratión metílico, entre otros (Rotterdam Convention, 2015; PAN, 2014; ONU, 2013).

Del cálculo de los indicadores de frecuencia se concentran la tasa de mortalidad infantil (TMF) presentes por región en el cuadro 2, para el corredor y para la zona no expuesta, por año y según la causa de la defunción.

Los resultados para la causa de anencefalia (Q00) y los valores de las TMF más altos se concentran en los municipios del corredor florícola, pues aun cuando las tasas van en disminución, éstas son superiores a las presentadas por los municipios de la zona no expuesta. Según el comportamiento lineal, de las dos causas analizadas, la anencefalia presenta las tasas más altas (gráfica 1), siguiendo el mismo patrón de
Gráfica 1. Comportamiento temporal de las TMF en el corredor florícola del Estado de México 1998-2013.

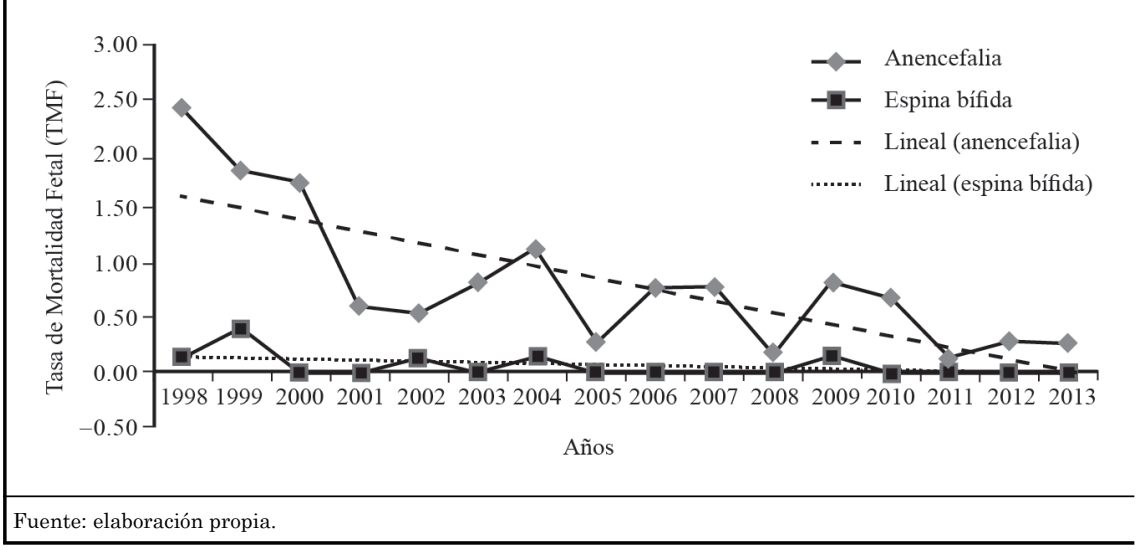


comportamiento a nivel nacional, donde es el tipo más común en los defectos del tubo neural (Ramírez-Espitia et al., 2003).

Para la causa de espina bífida (Q05), la TMF presenta una tendencia temporal en la que los registros anuales para el corredor florícola superan el valor de las tasas de la zona donde la población tiene actividades económicas diferentes a las agrícolas. En los municipios donde hay un mínimo de actividad económica agrícola, hay frecuencia en reporte de casos; sin embargo, sus tasas están por debajo del valor de las tasas de los municipios expuestos.

Las TMI, tanto por la causa de anencefalia como espina bífida, señalan valores superiores para el corredor florícola aunque con menor frecuencia de tasas, en comparación con los municipios no expuestos que reportan tasas siempre por debajo de una defunción infantil por cada 1000 habitantes. De acuerdo con

Gráfica 2. Comportamiento temporal de la tasa de mortalidad infantil (TMI) en el corredor florícola.

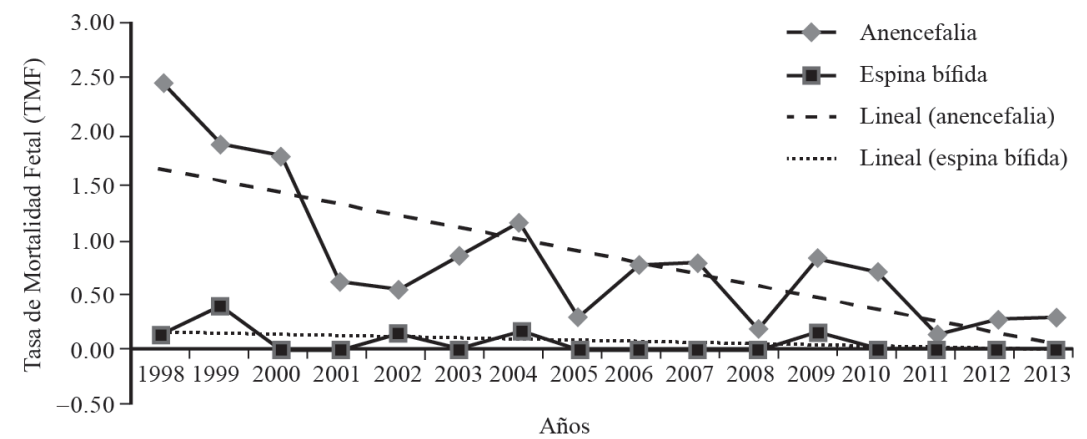

Fuente: elaboración propia.

Cuadro 2. Comportamiento de las medidas de frecuencia en el corredor por causa y año.

\begin{tabular}{|c|c|c|c|c|c|c|c|c|}
\hline Año & $\begin{array}{l}\text { TMF-Q00 } \\
\text { Exp. }\end{array}$ & $\begin{array}{l}\text { TMF-Q00 } \\
\text { No Exp. }\end{array}$ & $\begin{array}{c}\text { TMF-Q05 } \\
\text { Exp. }\end{array}$ & $\begin{array}{l}\text { TMF-Q05 } \\
\text { No Exp. }\end{array}$ & $\begin{array}{l}\text { TMI-Q00 } \\
\text { Exp. }\end{array}$ & $\begin{array}{l}\text { TMI-Q00 } \\
\text { No Exp. }\end{array}$ & $\begin{array}{l}\text { TMI-Q05 } \\
\text { Exp. }\end{array}$ & $\begin{array}{l}\text { TMI-Q05 } \\
\text { No Exp. }\end{array}$ \\
\hline 1998 & 2.41 & 0.49 & 0.13 & 0.05 & 0.29 & 0.13 & 1.18 & 0.08 \\
\hline 1999 & 1.84 & 0.63 & 0.39 & 0.06 & 0.14 & 0.21 & 1.15 & 0.09 \\
\hline 2000 & 1.74 & 0.43 & 0 & 0 & 0.28 & 0.09 & 0.71 & 0.08 \\
\hline 2001 & 0.61 & 0.40 & 0 & 0.03 & 0.14 & 0.08 & 0.29 & 0.06 \\
\hline 2002 & 0.54 & 0.24 & 0.13 & 0 & 0.15 & 0.18 & 0.30 & 0.02 \\
\hline 2003 & 0.83 & 0.27 & 0 & 0.02 & 0 & 0.12 & 0 & 0.06 \\
\hline 2004 & 1.11 & 0.09 & 0.14 & 0.03 & 0 & 0.13 & 0 & 0.06 \\
\hline 2005 & 0.29 & 0.12 & 0 & 0.02 & 0 & 0.09 & 0.17 & 0.07 \\
\hline 2006 & 0.75 & 0.18 & 0 & 0 & 0 & 0.09 & 0 & 0 \\
\hline 2007 & 0.76 & 0.17 & 0 & 0.01 & 0 & 0.08 & 0 & 0.05 \\
\hline 2008 & 0.19 & 0.28 & 0 & 0.02 & 0.18 & 0.05 & 0.18 & 0.01 \\
\hline 2009 & 0.82 & 0.10 & 0.14 & 0.03 & 0 & 0.09 & 0 & 0.01 \\
\hline 2010 & 0.68 & 0.19 & 0 & 0.01 & 0 & 0.06 & 0.18 & 0.01 \\
\hline 2011 & 0.13 & 0.11 & 0 & 0.05 & 0 & 0.04 & 0.19 & 0.03 \\
\hline 2012 & 0.27 & 0.16 & 0 & 0.03 & 0 & 0.03 & 0 & 0 \\
\hline 2013 & 0.27 & 0.10 & 0 & 0.04 & - & - & - & - \\
\hline
\end{tabular}

Nota: donde Exp.: zona expuesta y No Exp.: zona no expuesta. TMF calculadas por 1000 nacidos vivos y TMI por 1000 menores de un año de edad; No hay TMI para 2013 por falta de datos de población menor a 1 año de edad.

Fuente: elaboración con datos del SINAIS, 2016a; SINAIS, 2016b; SINAIS, 2016c la gráfica 2 la espina bífida es la que presenta los valores de frecuencia más altos.

La gráfica 3 presenta los municipios con las tasas más altas y de mayor frecuencia. Para el caso de mortalidad fetal por anencefalia, los más afectados son Tenancingo, Villa Guerrero y Zumpahuacán, que coinciden con los de mayor producción florícola según el Censo Agropecuario (INEGI, 2009). Se observa además en el comportamiento temporal, la tendencia lineal en descenso para Tenancingo y Villa Guerrero; sin embargo, en Zumpahuacán la TMF no ha disminuido a través del tiempo, sino que se han mantenido presentando una ligera tendencia al ascenso, situación de riesgo para la población. Una posible razón obedece a la mejor cobertura en servicios médicos para Tenancingo con trece unidades de consulta externa y dos hospitales generales, y Villa Guerrero doce unidades de consulta externa y un hospital integral comunitario, en comparación con Zumpahuacán que solo cuenta con seis unidades de consulta externa (Secretaría de Salud, 2010).

La población infantil afectada por espina bífida fue mayor para los municipios de Coatepec Harinas, Tenancingo, Zumpahuacán y Villa Guerrero; sin embargo, en el seguimiento temporal de 1998 a 2012 se observa una disminución en la tendencia lineal (gráfica 4). 
Al comparar las TMF y las TMI se ha identificado que la situación del área geográfica del corredor florícola respecto a la mortalidad fetal e infantil está relacionada con la presencia de malformaciones congénitas de anencefalia y espina bífida, donde la ocupación de los padres de los infantes está en el sector agrícola, lo que favorece la exposición continua a los plaguicidas, asociada a las defunciones fetales e infantiles.

De los indicadores de asociación, se obtuvo la razón de momios (odds ratio) y fracción atribuible para cada una de las tasas de mortalidad por causa a partir de las zonas consideradas como expuestas (municipios del corredor) y las no expuestas al factor de riesgo (cuadro 3).

De acuerdo con el indicador de razón de momios, en la zona expuesta se presenta 3.44 veces más casos de mortalidad fetal para la causa de anencefalia (Q00) que en la zona no expuesta. El valor más alto se presentó para la tasa de mortalidad infantil por la causa de espina bífida (Q05), que se presentó 7.11 veces más casos en la zona expuesta respecto a la no expuesta a los plaguicidas.

Respecto al riesgo atribuible (RA), el factor de riesgo considerado fue la exposición a plaguicidas por actividad ocupacional y por ubicación de residencias en el corredor. En el RA \% el valor más alto lo presentaron las defunciones fetales por anencefalia. El 65.2\% de la mortalidad fetal podría evitarse si el factor de riesgo se eliminará, es decir, si hay una disminución en la exposición a plaguicidas por parte de los padres.

Respecto a la espina bífida, $55 \%$ de las defunciones se podrían evitar o prevenirse si los padres no se hubieran expuesto a los plaguicidas. El valor más alto en las defunciones infantiles en menores de un año de edad lo presentó la espina bífida, donde $79.8 \%$ se pudo evitar o prevenirse si se eliminará el factor de exposición a plaguicidas.

Para la causa de defunciones por anencefalia, la asociación resultó negativa respecto a los municipios considerados como no expuestos. Esto está directamente relacionado con el resultado del RR (Riesgo Relativo), donde esta causa presentó el valor más bajo.

\section{Conclusiones}

Los municipios de mayor afectación por ambas causas de mortalidad fueron Zumpahuacán, Villa Guerrero, Coatepec Harinas y Tenancingo, que corresponden con los de mayor producción florícola y aplicación de plaguicidas. Como parte del trabajo de campo, se identificaron las principales vías de exposición a plaguicidas por los trabajadores dedicados a la floricultura a través de sus prácticas culturales como el consumo de alimentos

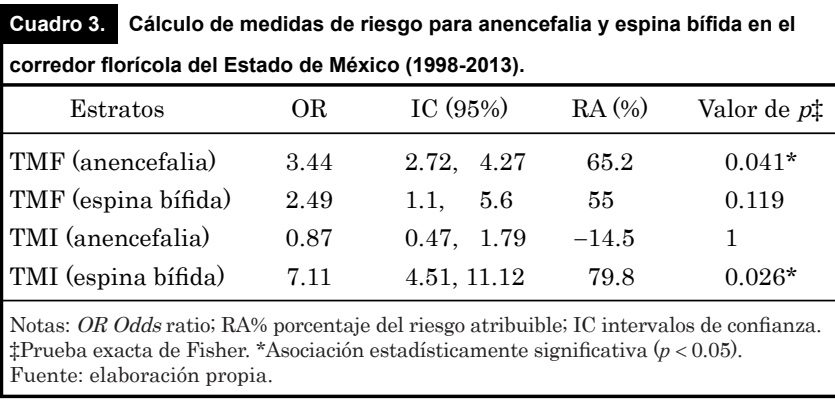

Gráfica 3. Comportamiento temporal de la tasa de mortalidad fetal (TMF) por anencefalia por municipio según el grado de afectación.

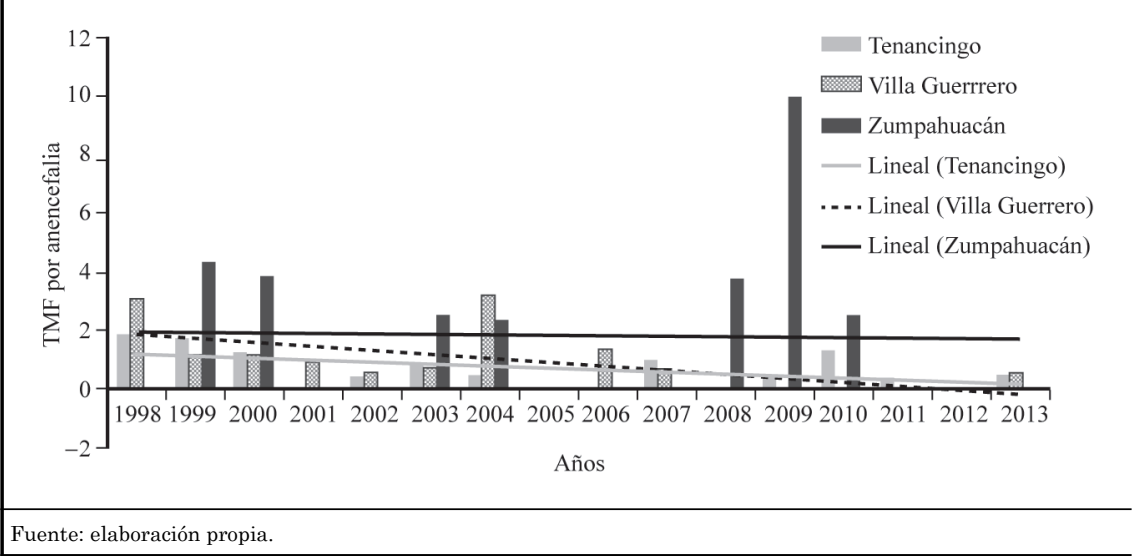

Gráfica 4. Comportamiento temporal de la tasa de mortalidad infantil (TMI) por espina bífida por municipio según el grado de afectación.

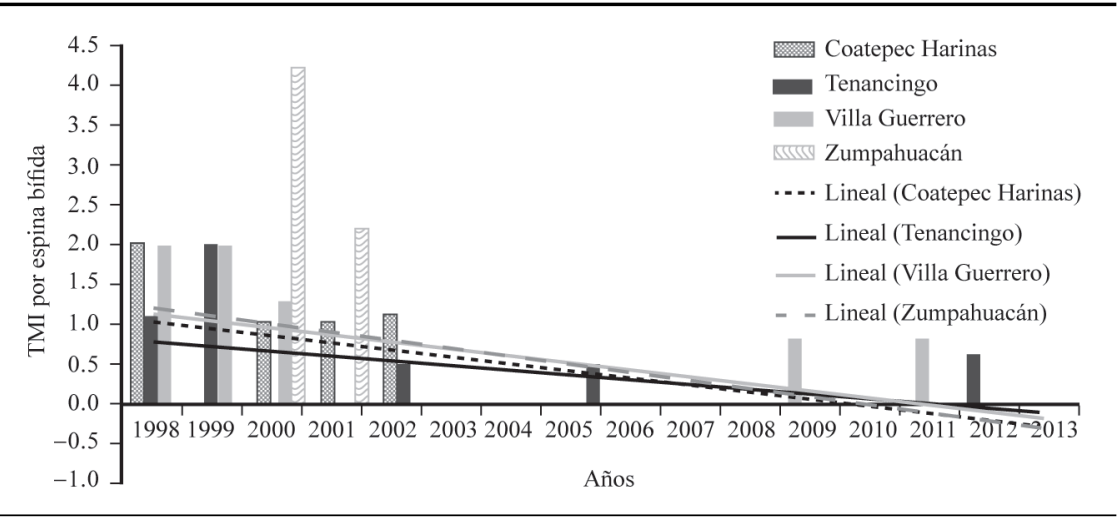

Fuente: elaboración propia. 
en el área de cultivo y uso continuo de ropa contaminada como las principales vías de exposición a plaguicidas.

Los resultados de los indicadores muestran una posible asociación entre la exposición a plaguicidas por actividad ocupacional florícola, y la mortalidad fetal e infantil por malformaciones congénitas.

Por lo tanto se sugiere, para estudios posteriores análisis más detallados como los experimentales, de cohorte o casos y controles que permitan corroborar la hipótesis de relación entre exposición y presencia de estas malformaciones congénitas. Identificar la asociación de la mortalidad por espina bífida y anencefalia con la exposición a los plaguicidas en el corredor florícola del Estado de México permitirá sugerir mejores políticas públicas de salud para la prevención a fin de disminuir la mortalidad por estas causas. Aun cuando su etiología es multifactorial, las prácticas en el manejo de plaguicidas están fuertemente vinculadas con estos efectos (Yang, 2014; Chen, 2008; Rull et al., 2006).

\section{Análisis prospectivo}

La actividad florícola en la región sur del Estado de México es de gran importancia tanto sociocultural como económica, basta con saber que genera más de 70 mil empleos, con una derrama de 4500 millones de pesos anualmente y exporta cerca de 30 millones de dólares. Sin embargo, el impulso no ha ido en paralelo con el buen uso y manejo de los plaguicidas. Durante el trabajo de campo se observó que son muy pocos los invernaderos tecnificados con uso de buenas prácticas en el manejo de plaguicidas. En este aspecto, la mayoría de los cultivos, tanto cerrados como abiertos, incurren en cuidados mínimos para los trabajadores, incluso utilizando plaguicidas prohibidos y restringidos a lo largo del país y que por tanto implican un mayor riesgo para la salud humana.
Desde el punto de vista geográfico el corredor florícola se encuentra rodeado de un sistema montañoso que propicia por efectos del viento y gravedad de agua que los residuos tóxicos de los plaguicidas que se aplican en las partes altas (Villa Guerrero, Coatepec, Malinalco y Tenancingo) sean transportados hacia las zonas bajas en el municipio de Zumpahuacán, lo que podría provocar que se vea mayormente afectado dado que fue el único municipio del corredor florícola que presentó un incremento temporal en las tasas de mortalidad fetal, por lo que es importante que los gobiernos del corredor florícola se coordinen desde un enfoque ecosistémico para el bienestar de la población.

La presente investigación contribuye a brindar información precisa para el sector salud de cómo los habitantes de la región están siendo afectados por los plaguicidas para así tomar medidas correctivas y preventivas. De acuerdo con los resultados obtenidos podrían evitar $65 \%$ de la mortalidad fetal por anencefalia y cerca de $80 \%$ de la mortalidad infantil de espina bífida.

Asimismo es importante una mayor regulación desde el marco legal el uso y venta de agroquímicos, retira los prohibidos por su alta toxicidad, y dar apertura a los orgánicos. Cabe mencionar que, aunque en la actualidad se está impulsando a nivel nacional el empleo de la agricultura orgánica, en la zona se pudo observar la escasez de este tipo de productos.

Para finalizar, se requiere identificar la dinámica de las vías o rutas de exposición a los plaguicidas como punto de partida para diseñar, proponer y ejecutar una gestión de estrategias que permita vincular la participación de las entidades gubernamentales, educativas y de la población en general, a fin de disminuir el riesgo tanto de muerte como de enfermedad por causas atribuibles a los plaguicidas en los próximos años.
Argimon J, Jiménez J. (2013). Métodos de investigación clínica y epidemiológica. $4^{\mathrm{a}}$ ed. Elsevier. Barcelona, España.

Benítez-Leite, S. Macchi, M. Acosta, M. (2009). Malformaciones congénitas asociadas a agrotóxicos. Revista Chilena de Pediatría, 80(4), 377-378. Consultado el 16 julio de 2016. Disponible en https://dx.doi. org/10.4067/S0370-41062009000400010

Blatter, B. M., Roeleveld, N., Bermejo, E.,
Martinez-Frias, M. L., Siffel, C., y Czeizel,

A. E. (2000). Spina bifida and parental occupation: results from three malformation monitoring programs in Europe. European Journal of Epidemiology, 16(4), 343-351. Consultado el 29 septiembre de 2016. Disponible en https:/ / www.ncbi.nlm.nih.gov/ pubmed/10959942

Brender, J. D., Felkner, M., Suarez, L., Canfield, M. A. y Henry, J. P. (2010). Maternal pes- ticide exposure and neural tube defects in Mexican Americans. Annals of Epidemiology, 20(1), 16-22. Consultado el 28 septiembre de 2016. Disponible en http://dx.doi. org/10.1016/j.annepidem.2009.09.011

Cevece (Centro Estatal de Vigilancia Epidemiológica y Control de Enfermedades). (2014). Proyecto: exposición a plaguicidas en habitantes de zonas florícolas del Estado de México. Gobierno del Estado de México. Secretaría de Salud. 
DGE (Dirección General de Epidemiología). (2012). Manual de procedimientos estandarizados para la vigilancia epidemiológica de los defectos del tubo neural. Secretaría de Salud. Consultado el 24 septiembre de 2014. Disponible en http://www.epidemiologia.salud.gob.mx/doctos/infoepid/ vig_epid_manuales/09_2012_Manual_DefTuboNeural_vFinal27sep12.pdf

Chen. C. (2008). Syndromes, disorders and maternal risk factors associated with neural tube defects (VI). Taiwanese Journal of Obstretics and Gynecology, 47(3), 267-275. Consultado el 25 julio de 2016. Disponible en http:/ / www.ncbi.nlm.nih.gov/pubmed/18603496

Espitia Rangel, E., Huerta Espino, J., Limón Ortega, A., Peña Bautista, R. J. y Villaseñor Mir, H. E. (2003). Calidad industrial de trigos harineros mexicanos para temporal. I. Comparación de variedades y causas de la variación. Revista Fitotecnia Mexicana, 26(4), 249-256. Consultado el 18 junio de 2016. Disponible en http://www.redalyc.org/ articulo.oa?id $=61026405$

FND (Financiera Nacional de Desarrollo). (2014). Panorama de los Ornamentos. Secretaría de Hacienda y Crédito Público. Consultado el 17 de agosto de 2015. Disponible en http://www.financierarural.gob.mx/informacionsectorrural/Panoramas/Ficha $\% 20$ Ornamentos.pdf

Goldman, L. Hopkins, J. (2004). Intoxicación por plaguicidas en niños. Información para la gestión y acción. PNUMA. Consultado el 18 julio de 2016. Disponible en http://www. unep.org/chemicalsandwaste/Portals/9/ Pesticides/ChildhoodPestPois_Sp.pdf

Goldman, L. (1997). New approaches for assessing the etiology and risks of developmental abnormalities from chemical exposure. Reprod Toxicol, 1997. p. 443-451.

Gómez-Alcalá A y Rascón-Pacheco, R. (2008). La mortalidad infantil por malformaciones congénitas en México: un problema de oportunidad y acceso al tratamiento. Revista Panamericana de Salud Pública, 24(45), 297-303. Consultado el 4 agosto de 2016. Disponible en http://www.scielosp.org/ pdf/rpsp/v24n5/01.pdf
González-Arias, C., Robledo-Marenco, M., Medina-Díaz, I., Velázquez-Fernández, J., Girón-Pérez, M., Quintanilla-Vega, B., Ostrosky-Wegman, P., Pérez-Herrera, N. y Rojas-García, A. (2010). Patrón de uso y venta de plaguicidas en Nayarit, México. Revista Internacional de Contaminación Ambiental 26(3), 221-228. Consultado el 13 julio de 2016. Disponible en http://www.scielo. org.mx/scielo.php?script=sci_arttext\&pid $=$ S0188-49992010000300005

Hernández-Antonio, A. y Hansen A. (2011). Uso de plaguicidas en dos zonas agrícolas de México y evaluación de la contaminación de agua y sedimentos. Revista Internacional de Contaminación Ambiental. 27(2), 115-127. Consultado el 14 julio de 2016. Disponible en http://www.scielo. org.mx/scielo.php?script=sci_arttext\&pid $=$ S0188-49992011000200003

Hernández-Ávila, D., Garrido-Latorre, M., y López-Moreno, S. (2000). Diseño de estudios epidemiológicos. Salud Pública de México, 42(2), 144-154. Consultado el 19 julio de 2016. Disponible en http://www.scielosp. org/pdf/spm/v42n2/2383.pdf

Hernández, M. (2007). Epidemiología. Diseño y análisis de estudio. Instituto Nacional de Salud Pública. México.

Hernández M. (2009). Epidemiología. Diseño y análisis de estudios. $1^{a}$ reimpresión. Instituto Nacional de Salud Pública. Médica Panamericana. México. D. F.

Herrera Tapia, F. (2006). Innovaciones tecnológicas en la agricultura empresarial mexicana: Una aproximación teórica. Revista Gaceta Laboral, 12(1), 93-119. Consultado el 29 septiembre de 2016. Disponible en http:/ / www.redalyc.org/pdf/336/33612105.pdf INEGI (Instituto Nacional de Estadística Geografía e Informática). (2013). Mujeres y bombres en México 2013. Instituto Nacional de las Mujeres. Consultado el 17 agosto de 2015. Disponible en http:/ / cedoc.inmujeres.gob. $\mathrm{mx} /$ documentos_download/101215.pdf

INEGI (Instituto Nacional de Estadística Geografía e Informática). (2011). Serie V Uso de Suelo y Vegetación 1:250 000, Carta E14-2 Ciudad de México. Descarga de datos vectoriales. Recursos Naturales. Consultado el 19 mayo de 2014. Disponible en http:// www.inegi.org.mx/geo/contenidos/recnat/ usosuelo/Default.aspx

INEGI (Instituto Nacional de Estadística Geografía e Informática). (2010). Censo de Población y Vivienda. Consultado el 17 agosto de 2015. Disponible en http://www.inegi. org.mx/est/contenidos/proyectos/ccpv/ cpv2010/Default.aspx

INEGI (Instituto Nacional de Estadística Geografía e Informática). (2009). Censo Agropecuario 2007, VIII Censo Agrícola, Ganadero y Forestal. Consultado el 6 de junio de 2014. Disponible en http://www.inegi. org.mx/est/contenidos/proyectos/Agro/ ca2007/Resultados_Agricola/default.aspx

Jewell, N. (2004). Statistics for epidemiology. Florida: Chapman \& Hall/CRC. Consultado el 23 agosto de 2016. Disponible en http:// file.zums.ac.ir/ebook/498-Statistics $\% 20$ for $\% 20$ Epidemiology-Nicholas $\% 20$ P. $\% 20$ Jewell-1584884339-CRC-2003-352-\$94.pdf

Karam-Quiñones, C. (2002). Los agroquimicos: una perspectiva jurídica-ambiental. Análisis del caso de Sinaloa. Culiacán: Colegio de Sinaloa.

Karam, M. Á., Ramírez, G., Bustamante, L. P., Galván, J. M., (2004). Plaguicidas y salud de la población. CIENCIA ergo-sum, 11(3), 246-254. Universidad Autónoma del Estado de México. Toluca, México. Consultado el 14 agosto de 2015. Disponible en http:// www.redalyc.org $/$ articulo.oa?id $=10411304$ Leyva Morales, J. B., García de la Parra, L. M., Bastidas Bastidas, P. D. J., Astorga Rodríguez, J. E., Bejarano Trujillo, J., Cruz Hernández, A., Martínez Rodríguez, I. E. y Betancourt Lozano, M. (2014). Uso de plaguicidas en un valle agrícola tecnificado en el noroeste de México. Revista Internacional de Contaminación Ambiental, 30(3), 247-261. Consultado el 15 julio de 2016. Disponible en http://www.scielo. org.mx/scielo.php?script=sci_arttext\&pid $=$ S0188-49992014000300002

Mancebo-Hernández, A., González-Rivera A., Díaz-Omaña, L., López-Alquicira, M., Domínguez-Viveros, W. y Serrano-Sierra, A. (2008). Defectos del tubo neural. Panorama 
epidemiológico en México (I de II). Acta

Pediátrica de México, 29(1), 41-47. Consultado el 17 agosto de 2015. Disponible en http:// www.medigraphic.com/pdfs/actpedmex/ apm-2008/apm081i.pdf

Moreno-Altamirano, A., López-Moreno, S. y Corcho-Verdugo, A. (2000). Principales medidas en epidemiología. Salud Pública de México, 42(4), 337-348. Consultado el 14 marzo de 2016. Disponible en http:/ / www. scielosp.org/pdf/spm/v42n4/2882.pdf

Muñoz Piña, C. y Ávila Forcada, S. (2005). Los efectos de un impuesto ambiental a los plaguicidas en México. Gaceta Ecológica, 74(1), 43-53. Consultado el 6 julio de 2016. Disponible en http://www.redalyc.org/toc. oa?id $=539$ \&numero $=2360$

OMS (2007). Un nuevo informe de la OMS aborda la salud ambiental de niños. Centro de Prensa. Consultado el 3 marzo de 2016. Disponible en http://www.who.int/mediacentre/news/notes/2007/np27/es/

ONU (Organización de las Naciones Unidas).

(2013). FAO urge eliminar plaguicidas peligrosos en paises en desarrollo. Centro de Noticias oNU. Consultado el 17 junio de 2015. Disponible en http://www.un.org/spanish/News/story.asp?NewsID=27073\#. VYHLTbtRHIU

ops (Organización Panamericana de la Salud) y oms (Organización Mundial de la Salud). (2008). Clasificación estadística Internacional de enfermedades y problemas relacionados con la salud (CIE-10). Décima revisión. Volumen 3. Consultado el 14 marzo de 2016. Disponible en http://ais.paho.org/ classifications/Chapters/pdf/Volume3.pdf

Oviedo-Zúñiga, A. M., Karam-Calderón Miguel, A. y Rodríguez García, C. (2003). Percepción de riesgo por el uso de plaguicidas en niños escolares, Villa Guerrero, Estado de México. retel. Revista de Toxicología en línea, 11, 23-46. Consultado el 17 agosto de 2015. Disponible en http://www.sertox.com.ar/ retel/n11/04.pdf

PAN (Red Internacional de Acción contra Plaguicidas). (2014). Lista de plaguicidas altamente peligrosos (lista de PAN de pap). Consultado el 28 junio de 2015.
Disponible en http://www.rap-al.org/ news_files/HHP\%20Lista $\% 20$ PAN $\% 20$ 2014\%20fin.pdf

Peña-Alonso, R., Venegas, C., Lozano, R., Kofman-Alfaro, S. y Queipo, G. (2005). Mortalidad por defectos al nacimiento. Boletín Médico del Hospital Infantil de México, 62(4), 294-304. Consultado el 17 agosto de 2015. Disponible en http://www.medigraphic. com/pdfs/bmhim/hi-2005/hi054i.pdf

Plengue-Tellechea, F., Sierra-Fonseca, J. y Castillo-Sosa, Y. (2007). Riesgos a la salud humana causados por plaguicidas. Tecnociencia, 1(3), 4-6. Consultado el 18 julio de 2016. Disponible en http://tecnociencia. uach.mx/numeros/numeros/v1n3/data/ cientifico_sociedad.pdf

Ramírez-Espitia, J., Benavides, F., LacasañaNavarro, M., Martínez, J., García, A. y Benach, J. (2003). Mortalidad por defectos del tubo neural en México, 1980-1997. Salud Pública de México, 45(5), 356-364. Consultado el 18 julio de 2016. Disponible en http://bvs.insp.mx/rsp/_files/File/2003/ num_5/455_4_mortalidad.pdf

Rotterdam Convention. (2015). Notificaciones de Medidas Reglamentarias Firmes. ONU-UNEP-FAO. Consultado el 20 junio de 2015. Disponible en http://www.pic.int/Procedimientos/ NotificacionesdeMedidasReglamentariasFirmes/BasedeDatos/tabid/2014/language/es-CO/Default.aspx

Rull, R. P., Ritz, B. y Shaw, G. M. (2006). Neural tube defects and maternal residential proximity to agricultural pesticide applications. American Journal Epidemiology, 163, 743-753. Consultado el 25 julio de 2016. Disponible en http://www.ncbi.nlm.nih.gov/pubmed/16495467

Sánchez, J., Pacheco, F. (2009). Floricultura, en La diversidad Biológica del Estado de México pp. 291-297. Toluca: Gobierno del Estado de México. Consultado el 18 julio de 2016. Disponible en http://www.biodiversidad. gob.mx/region/EEB/pdf/FinalBiodivEdoMexweb.pdf

Samborn, M., Bassil, K., Vakil, C., Kerr, K. y Ragan, K., (2012). Systematic review of pesticide health effects. Ontario College of
Family Physicians (OCFP). Consultado el 29 septiembre de 2016. Disponible en http:// ocfp.on.ca/docs/pesticides-paper/2012systematic-review-of-pesticide.pdf

Secretaría de Salud. (2010). CLuES. Dirección General de Información en Salud (DGIS). Consultado el 4 abril de 2014. Disponible en http://www.sinais.salud.gob.mx

SINAIS (2016a). Bases de datos de Defunciones Generales 1998-2012. DGIS, Secretaría de Salud. Consultado el 14 marzo de 2016. Disponible en http://www.sinais.salud.gob.mx/basesdedatos/std_defunciones.html

SINAIS (2016b). Bases de datos de Muertes Fetales 1998-2012. DGIS, Secretaría de Salud. Consultado el 14 marzo de 2016. Disponible en http://www.sinais.salud.gob.mx/basesdedatos/std_muertesfetales.html

SINAIS (2016c). Bases de datos sobre Nacimientos 1998-2012. DGIS, Secretaría de Salud. Consultado el 14 marzo de 2016. Disponible en http://www.sinais.salud.gob.mx/basesdedatos/std_nacimientos.html

Stemp-Morlock, G. (2007). Reproductive health: Pesticides and anencephaly. Environmental Health Perspectives, 115(2), A78. Consultado el 29 septiembre de 2016. Disponible en https://www.ncbi.nlm.nih. gov/pmc/articles/PMC1817703/

Viqueira, C., Palerm. A. y Olivares, L. (1971). Manual de Campo del Antropólogo (versión esp.). Instituto Real de Antropología de la Gran Bretaña e Irlanda. México: Universidad Iberoamericana.

WorldClim. (2005). Temperatura media de 19502000, Resolución de 30 segundos de arco (1 km). Descarga de Datos. Consultado el 24 abril de 2014. Disponible en http://www. worldclim.org/

Yang, W., Carmichael, S. L., Roberts, E. M., Kegley, S. E., Padula, A. M., English, P. B., Shaw, G. M. (2014). Residential agricultural pesticide exposures and risk of neural tube defects and orofacial clefts among offspring in the San Joaquin Valley of California. American Journal of. Epidemiology, 18, 1-9. Consultado el 25 julio de 2016. Disponible en http://www.ncbi.nlm.nih.gov/pubmed $/ 24553680$ 